\title{
Colletotrichum aenigma Associated with Apple Bitter Rot on Newly Bred cv. RubyS Apple
}

*Corresponding author

Tel: +82-53-950-5760

Fax: +82-53-950-6758

E-mail: heeyoung@knu.ac.kr

ORCID

https://orcid.org/0000-0002-4254-3367

Received May 14, 2021

Revised June 28, 2021

Accepted June 28, 2021

\author{
Seung-Yeol Lee ${ }^{1,2}$, Leonid N. Ten ${ }^{1}$, Jung-Joo Ryu ${ }^{1}$, In-Kyu Kang ${ }^{1}$, and Hee-Young \\ Jung ${ }^{1,2^{*}}$ (D) \\ ${ }^{1}$ College of Agriculture and Life Sciences, Kyungpook National University, Daegu 41566, Korea \\ ${ }^{2}$ Institute of Plant Medicine, Kyungpook National University, Daegu 41566, Korea
}

\begin{abstract}
The abnormal brown sunken lesions were observed on cv. RubyS apple fruits in an orchard located in Gunwi, Gyeongbuk province, Korea. The primary observed symptoms such as small round sunken lesions and small black dots on the symptomatic area were different from the reported apple diseases. The affected apple fruits were sampled and subjected to isolation of the causal agent. Cultural and morphological characteristics of isolated fungal strain, designated KNUF-20GWA4, were similar to that of Colletotrichum spp. Based on multilocus sequence analyses using internal transcribed spacer regions and partial sequences of $\beta$-tubulin, glyceraldehyde-3-phosphate dehydrogenase, chitin synthase, and actin genes, strain KNUF-20GWA4 showed 99.2-100\% similarities with C. aenigma ICMP 18608 and the isolate clustered together with several other strains of this species in the phylogenetic tree. To our knowledge, this is the first report of bitter rot on apple fruits caused by C. aenigma.
\end{abstract}

Keywords: Apple, Bitter rot, Colletotrichum aenigma, cv. RubyS
Apples (Malus domestica) are cultivated worldwide, with production reaching approximately 87 million tons in 2019 (FAOSTAT, 2019). In Korea, the area used for apple cultivation is approximately 30,000 ha, and about $90 \%$ of apple cultivars are Fuji and Hongro (Choi et al., 2018). A new apple cultivar, 'RubyS', was developed by the National Institute of Horticultural Science at the Rural Development Administration and registered in 2015. The main characteristics of cv. RubyS are high resistance to apple bitter rot and longer storability at room temperature than other apple cultivars (Kwon et al., 2019). Due to the demand for medium-sized apples in Korea, the cv. RubyS cultivation area has increased to approximately $105 \mathrm{ha}$. The damage caused by various fungal diseases is considered to be one of the main factors limiting the commercial production of apples. It is well known that

Research in Plant Disease

pISSN 1598-2262, elSSN 2233-9191

www.online-rpd.org the key to effective disease management starts with the correct diagnosis of the causal agent. In this study, a fungal pathogen was isolated from the affected cv. RubyS apple fruits and it was identified as a new causal agent of apple bitter rot in Korea.

In September 2020, abnormal brown spot symptoms were observed on cv. RubyS apple fruits in an orchard located in Gunwi, Gyeongbuk province, Korea. The estimated infection rate was observed around $5 \%$ on the surveyed orchard. The affected areas were light to dark brown inside, surrounded a dark ring. A solid symptomatic lesion, circular or irregular in shape, and small black dots were observed on the symptomatic area (Fig. 1A-D). These symptomatic features were distinct from the symptoms observed on infected apple fruits that are typical of fungal diseases such as bitter rot or white rot (Uhm, 2010). The causal agent was isolated from the abnormal brown spots by wiping the surface of an affected apple with $70 \% \mathrm{EtOH}$. Then, a thin layer of the diseased peel was removed using a sterilized blade, and the diseased tis- 


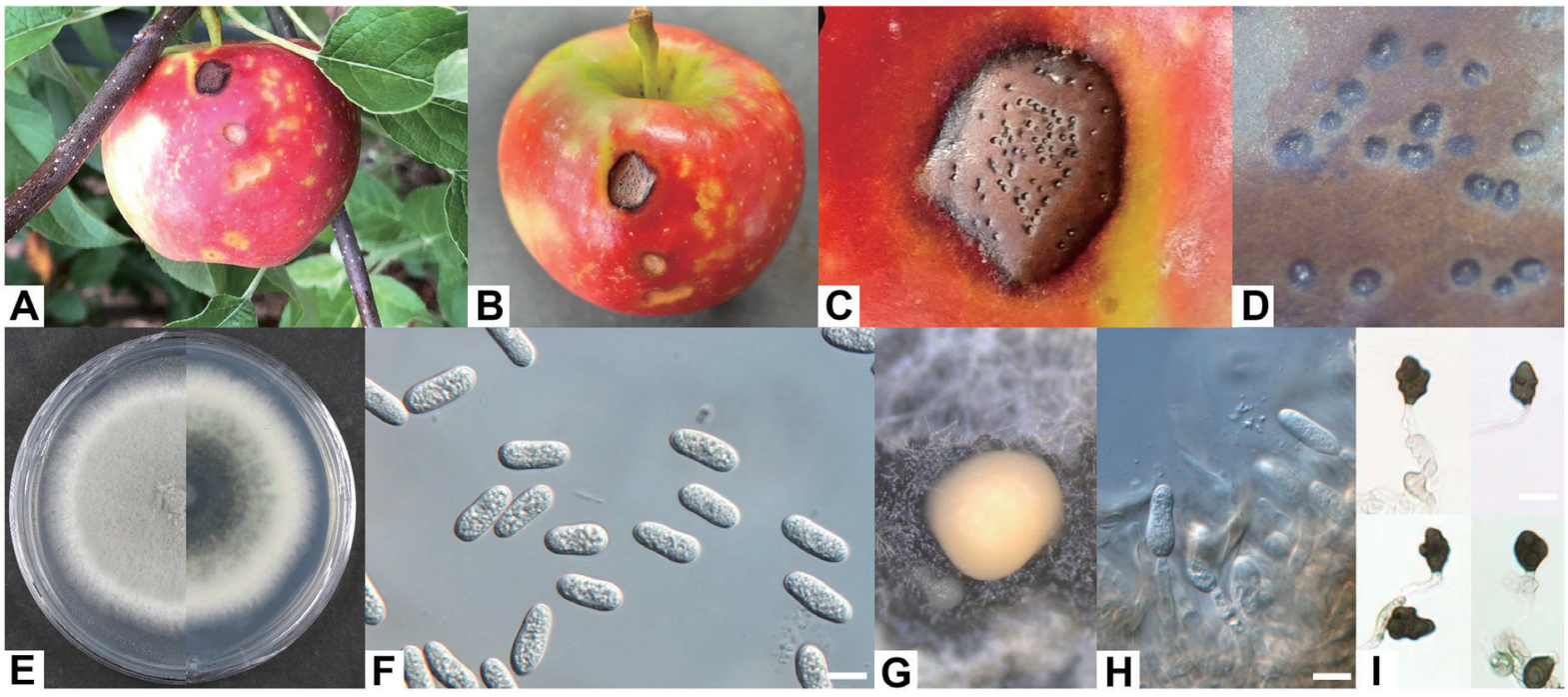

Fig. 1. Abnormal brown spot symptoms observed on cv. RubyS apple fruits and morphological characteristics of the isolate. (A-D) Photographs showing abnormal brown sunken lesion on RubyS diseased apple fruits collected from Gunwi in 2020. (A) Infected RubyS apple collected from the field. (B) Close-up view of the infected apple in A. (C) Symptomatic area on a RubyS apple. (D) Black dots on the symptomatic region. (E) Five-day-old fungal culture on potato dextrose agar (PDA). (F-l) Light microscopy showing conidia (F), conidiomata (G), a conidiogenous cell $(\mathrm{H})$, and appressoria (I) from the PDA culture. Scale bars=10 $\mu \mathrm{m}(\mathrm{F}-\mathrm{I})$.

sues were directly transferred onto potato dextrose agar (PDA; Difco, Detroit, MI, USA) and incubated at $25^{\circ} \mathrm{C}$. After 3 days, white mycelium was transferred onto new PDA plates and formed colonies that were used to further identify the strain, designated KNUF-20GWA4. The fungal isolate was identified by extracting genomic DNA from a 5-day-old fungal culture grown on PDA medium using a HiGeneGenomic DNA Prep Kit (BIOFACT, Daejeon, Korea), as per the manufacturer's protocol. The internal transcribed spacer (ITS) regions, including ITS1, 5.8S, and ITS2, were amplified using the ITS1/ ITS4 (White et al., 1990). The amplified PCR products were purified with ExoSAP-IT PCR Product Cleaning Reagent (Thermo Fisher Scientific, Waltham, MA, USA) then requested the sequencing analysis (SolGent, Daejeon, Korea). A sequence of $581 \mathrm{bp}$ was obtained from the ITS regions. A BLAST search in the National Center for Biotechnology Information database revealed similarities of $100 \%$ between the ITS regions of KNUF-20GWA4 and several strains belonging to Colletotrichum aenigma, C. siamense, and C. gloeosporioides, indicating that the ITS sequence was unsuitable for precise identification of the isolate.

Recently, various combinations of molecular markers were used for multilocus phylogenetic analysis to distinguish closely related taxa in C. gloeosporioides species com- plexes (Fu et al., 2019; Oo et al., 2017; Wang et al., 2021). Thus, we used this approach to amplify the $\beta$-tubulin ( $\beta$-TUB), glyceraldehyde-3-phosphate dehydrogenase (GAPDH), chitin synthase (CHS), and actin (ACT) genes of strain KNUF20GWA4 according to previous reports (Carbone and Kohn, 1999; Glass and Donaldson, 1995; Guerber et al., 2003). The amplification of $\beta-T U B, G A P D H, C H S$, and ACT loci yielded fragments of 580,266, 270, and $254 \mathrm{bp}$, respectively. All the gene sequences were registered in NCBI with the following GenBank (accession numbers MZ068034, LC630937-LC630940, respectively.). Based on the partial gene sequences of the four molecular markers, strain KNUF20GWA4 showed $99.2-100 \%$ similarities with those of $C$. aenigma ICMP $18608^{\top}$. To confirm the closest relationship, phylogenetic analysis using concatenated sequences of the ITS regions, $\beta$-TUB, GAPDH, CHS, and ACT genes were performed. The sequences of allied species were retrieved from NCBI GenBank (Table 1) and a phylogenetic tree was constructed using the maximum-likelihood method as implemented in MEGA7 software (Kumar et al., 2016). Strain KNUF-20GWA4 clustered together with C. aenigma IY1059, C. aenigma GRAP12, and C. aenigma GRAP07 isolated in Korea (Choi et al., 2017; Kim et al., 2021) and two other strains of C. aenigma ICMP 18608 and ICMP 18686 in a monophy- 
Table 1. Colletotrichum species used in this study for phylogenetic analysis and their GenBank accession numbers

\begin{tabular}{|c|c|c|c|c|c|c|}
\hline \multirow{2}{*}{ Species } & \multirow{2}{*}{ Strain no. } & \multicolumn{5}{|c|}{ GenBank accession no. } \\
\hline & & ITS & $\beta$-TUB & GAPDH & CHS & АCT \\
\hline Colletotrichum aenigma & ICMP 18686 & JX010243 & JX010390 & JX009913 & JX009789 & JX009519 \\
\hline Colletotrichum aenigma & ICMP 18608 & $J X 010244$ & JX010389 & JX010044 & JX009774 & JX009443 \\
\hline Colletotrichum aenigma & IY1059 & KY820893 & KY820891 & KY820889 & KY820888 & KY820892 \\
\hline Colletotrichum aenigma & GRAP12 & LC586813 & LC586816 & LC586819 & LC586822 & LC586825 \\
\hline Colletotrichum aenigma & GRAP7 & LC586811 & LC586814 & LC586817 & LC586820 & LC586823 \\
\hline Colletotrichum aenigma & KNUF-20GWA4 & MZ068034 & LC630940 & LC630939 & LC630938 & LC630937 \\
\hline Colletotrichum alienum & CBS 133930 & KC297076 & KC297096 & KC297000 & KC296982 & KC296938 \\
\hline Colletotrichum alienum & ICMP 12071 & JX010251 & JX010411 & JX010028 & JX009882 & JX009572 \\
\hline Colletotrichum alienum & CBS 111982 & KC297069 & KC297091 & KC296998 & KC296975 & KC296932 \\
\hline Colletotrichum fructicola & ICMP 18581 & JX010165 & JX010405 & JX010033 & JX009866 & FJ907426 \\
\hline Colletotrichum fructicola & CBS 125397 & JX010173 & JX010409 & JX010032 & JX009874 & JX009581 \\
\hline Colletotrichum musae & IMI 52264 & JX010142 & JX010395 & JX010015 & JX009815 & JX009432 \\
\hline Colletotrichum musae & CBS 116870 & JX010146 & HQ596280 & JX010050 & JX009896 & JX009433 \\
\hline Colletotrichum nupharicola & CBS 472.96 & JX010188 & JX010399 & JX010031 & JX009836 & JX009582 \\
\hline Colletotrichum nupharicola & CBS 470.96 & JX010187 & JX010398 & JX009972 & JX009835 & JX009437 \\
\hline Colletotrichum nupharicola & CBS 469.96 & JX010189 & JX010397 & JX009936 & JX009834 & JX009486 \\
\hline Colletotrichum siamense & CBS 113199 & KC297066 & KC297090 & KC297008 & KC296985 & KC296930 \\
\hline Colletotrichum siamense & CBS 112983 & KC297065 & KC297100 & KC297007 & KC296984 & KC296929 \\
\hline Colletotrichum boninense & CBS 123755 & JX010292 & JQ005588 & JX009905 & JX009827 & JX009583 \\
\hline
\end{tabular}

The isolated strain is shown in bold.

ITS, internal transcribed spacer; $\beta$-TUB, $\beta$-tubulin gene; GAPDH, glyceraldehyde 3-phosphate dehydrogenase gene; $C H S$, chitin synthases gene; ACT, actin gene.

letic clade with a high bootstrap value, strongly supporting their affiliation to the same species (Fig. 2).

Strain KNUF-20GWA4 was cultured for 7 days on PDA at $25^{\circ} \mathrm{C}$, and colony characteristics, such as color, shape, and size, were recorded. The colonies were floccose and white to gray with a white margin, with a dark green to gray center on reverse. The morphological characteristics were observed by light microscopy using a BX50 microscope (Olympus, Tokyo, Japan). The conidia were abundant and cylindrical with round ends, sometimes slightly curved, tapering toward 1 or both ends, and measured 14.921.1 $\times 5.7-8.5 \mu \mathrm{m}$ (mean, $18.6 \times 7.3 ; n=50$ ) The appressoria were brown to dark brown, clavate, crenate with lobes, and measured 9.2-17.7×5.2-8.8 $\mu$ m (mean, 12.0 $\times 7.5 ; n=30$ ) (Fig. $1 \mathrm{E}-\mathrm{I})$. These morphological and cultural characteristics of isolate KNUF-20GWA4 were in good agreement with those previously reported for C. aenigma (Wang et al., 2016; Weir et al., 2012), thus strongly supporting phylogenetic analysis results of KNUF-20GWA4 based on the above-mentioned five concatenated sequences.

Strain KNUF-20GWA4 was inoculated onto three healthy Cv. RubyS apple fruits to confirm its pathogenicity. The spore suspension for the inoculum was prepared after culturing the isolate for 15 days, and the conidial concentration was determined with a hemocytometer and adjusted to approximately $6.8 \times 10^{5}$ conidia/ $\mathrm{ml}$. The surface of a healthy apple fruit was wiped using $70 \% \mathrm{EtOH}$, and two lenticels were wounded using a sterilized needle. Then, paper disks containing $30 \mu \mathrm{l}$ of spore suspension were attached and sealed using foil, while sterilized water was used as a mock inocula- 


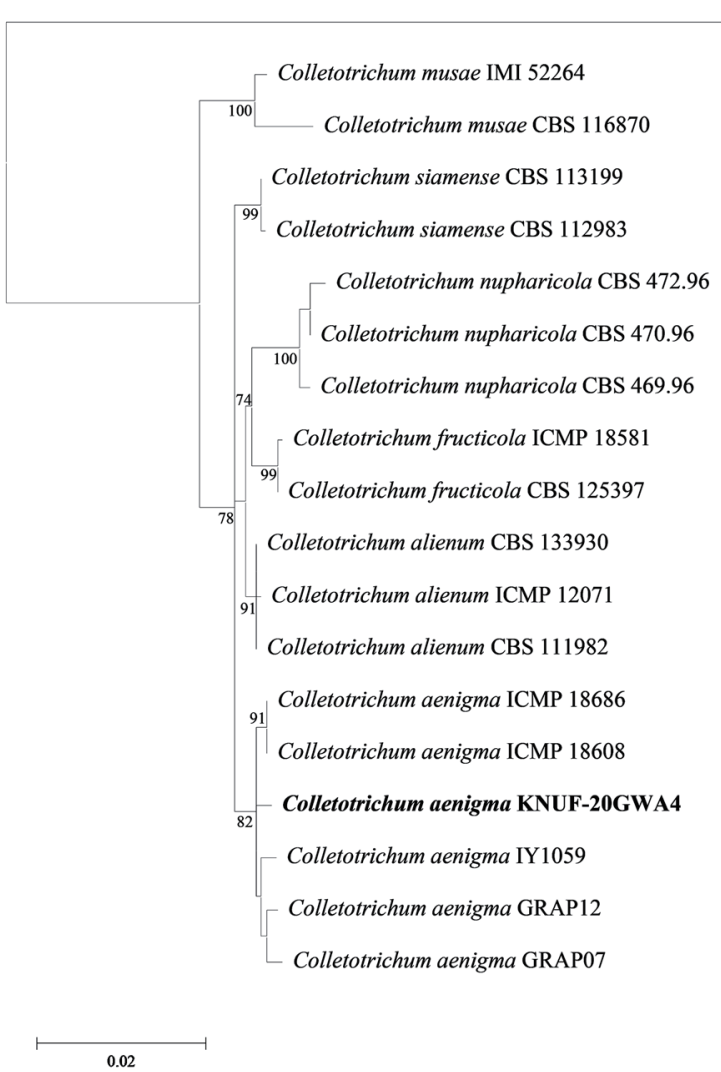

Colletotrichum boninense CBS 123755

Fig. 2. Maximum-likelihood phylogenetic tree, based on the concatenated sequences (ITS+ $\beta-T U B+G A P D H+C H S-1+A C T$ ), shows the phylogenetic position of Colletotrichum aenigma KNUF-20GW4 among the closest Colletotrichum species. The isolated strain is shown in bold. Bootstrap values (based on 1,000 replications) $>70 \%$ are shown at the branch points. Colletotrichum boninense CBS 123755 was used as the outgroup. Scale bar $=0.02$ substitutions per nucleotide position.

tion. Identical paper disks were also attached to unwounded lenticels and sealed using foil. After 14 days, brown spots with conidiomata were only observed on the wounded points on all the inoculated fruits, while no symptoms were observed on the unwounded and mock-inoculated parts (Fig. 3). The symptomatic lesions slowly increased in size and were solid, similar to the natural disease symptoms. The fungal strain was re-isolated and identified as C. aenigma from the symptomatic areas of each inoculated fruit (data not shown). Meanwhile, C. aenigma KNUF-20GWA4 was also inoculated on cv. Fuji apple fruits using the same method, however, no symptoms appeared after 14 days (data not

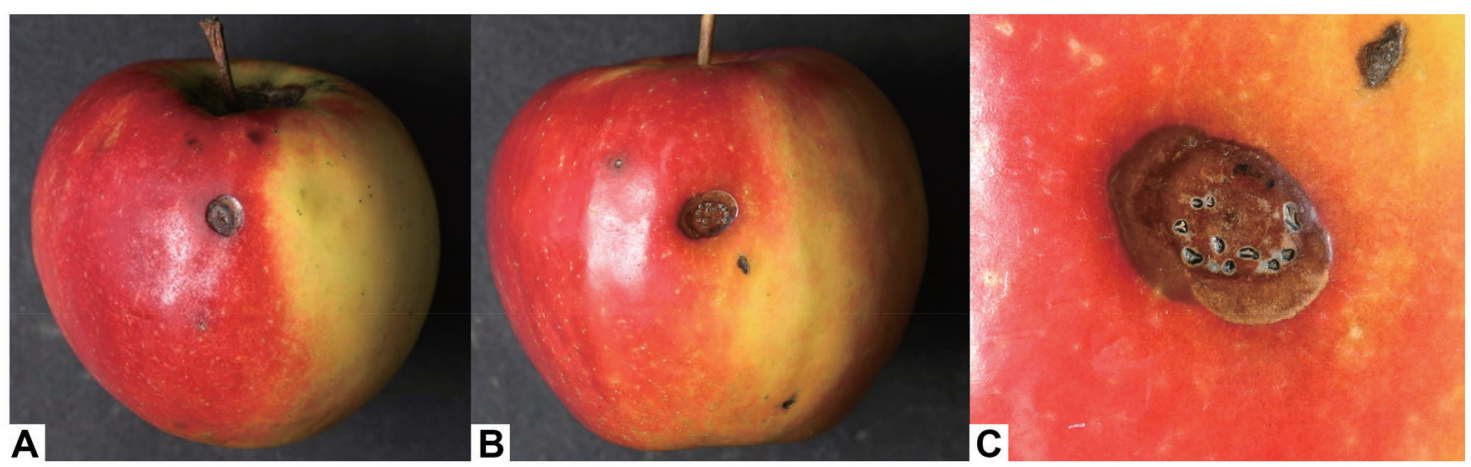

Fig. 3. Pathogenicity test results of Colletotrichum aenigma KNUF-20GWA4. (A, B) Symptoms on the inoculated cv. RubyS apple. (C) Enlarged picture of $B$ showing the conidiomata on the symptomatic area. 
shown).

C. aenigma has been reported as a pathogen associated with anthracnose on several hosts, including grape and Sedum kamtschaticum (Orange stonecrop) in Korea (Choi et al., 2017; Kim et al., 2021); grapevine, Asian pear, and walnut in China (Fu et al., 2019; Wang et al., 2021; Yan et al. 2015); avocado in Israel (Sharma et al., 2017); Synsepalum dulcificum (miracle fruit) in Japan (Truong et al., 2018); and Hylocereus undatus (dragon fruit) in Thailand (Meetum et al., 2015). However, this fungal pathogen has not been reported as the causal agent of bitter rot on apple fruits.

Until now, various Colletotrichum species have been reported on the apple fruits in Korea and causes significant economic losses in apple cultivation (Oo et al., 2018; Uhm, 2010). Although it was confirmed that the isolated C. aenigma has no pathogenicity on cv. Fuji apples in this study, however, it is necessary to confirm the pathogenicity on the different apple cultivars and the possibility of co-infection. To our knowledge, this is the first report of $C$. aenigma as the agent of apple bitter rot on apple cv. RubyS. Further studies are necessary to better define the host range of $C$. aenigma in Korea, the impact of the disease on yield, and control methods.

\section{Conflicts of Interest}

No potential conflict of interest relevant to this article was reported.

\section{Acknowledgments}

This research was supported by Kyungpook National University Research Fund, 2018.

\section{References}

Carbone, I. and Kohn, L. M. 1999. A method for designing primer sets for speciation studies in filamentous ascomycetes. Mycologia 91: 553-556.

Choi, D.-W., Kim, D.-C. and Lim, C.-R. 2018. Analysis of factors influencing cultivation area of apple cultivars. J. Korean Soc. Rural Plan. 24: 25-31.

Choi, H.-W., Lee, Y. K. and Hong, S. K. 2017. First report of Colletotrichum aenigma causing anthracnose on Sedum kamtschaticum in Korea. Plant Dis. 101: 2150.

FAOSTAT. 2019. Food and Agriculture Organization of the United
Nations. URL http://www.fao.org/ [30 May 2021].

Fu, M., Crous, P. W., Bai, Q., Zhang, P. F., Xiang, J., Guo, Y. S. et al. 2019. Colletotrichum species associated with anthracnose of $P y$ rus spp. in China. Persoonia 42: 1-35.

Glass, N. L. and Donaldson, G. C. 1995. Development of primer sets designed for use with the PCR to amplify conserved genes from filamentous ascomycetes. Appl. Environ. Microbiol. 61: 13231330.

Guerber, J. C., Liu, B., Correll, J. C. and Johnston, P. R. 2003. Characterization of diversity in Colletotrichum acutatum sensu lato by sequence analysis of two gene introns, mtDNA and intron RFLPs, and mating compatibility. Mycologia 95: 872-895.

Kim, J. S., Hassan, O. and Chang, T. 2021. First report of Colletotrichum aenigma causing anthracnose of grape in Korea. Plant. Dis. Advanced online publication. https://doi.org/10.1094/PDIS11-20-2458-PDN.

Kumar, S., Stecher, G. and Tamura, K. 2016. MEGA7: molecular evolutionary genetics analysis version 7.0 for bigger datasets. Mol. Biol. Evol. 33: 1870-1874.

Kwon, S.-I., Kim, J.-H., Kim, S.-A. and Kwon, Y.-S. 2019. 'RubyS', a small apple. HortScience 54: 2067-2069.

Meetum, P., Leksomboon, C. and Kanjanamaneesathian, M. 2015. First report of Colletotrichum aenigma and C. siamense, the causal agent of anthracnose disease of dragon fruit in Thailand. J. Plant Pathol. 97: 391-403.

Oo, M. M. and Oh, S.-K. 2017. New record of anthracnose caused by Colletotrichum liriopes on broadleaf Liriope in Korea. Korean J. Mycol. 45: 68-73.

Oo, M. M., Yoon, H.-Y., Jang, H. A. and Oh, S.-K. 2018. Identification and characterization of Colletotrichum species associated with bitter rot disease of apple in South Korea. Plant Pathol. J. 34: 480-489.

Sharma, G., Maymon, M. and Freeman, S. 2017. Epidemiology, pathology and identification of Colletotrichum including a novel species associated with avocado (Persea americana) anthracnose in Israel. Sci. Rep. 7: 15839.

Truong, H. H., Sato, T., Ishikawa, S., Minoshima, A., Nishimura, T. and Hirooka, Y. 2018. Three Colletotrichum species responsible for anthracnose on Synsepalum dulcificum (miracle fruit). Int. J. Phytopathol. 7: 89-101.

Uhm, J. Y. 2010. Reduced Fungicide Spray Program for Major Apple Diseases Korea. Agriculture and Horticulture Press, Anyang, Korea. $251 \mathrm{pp}$.

Wang, Y.-C., Hao, X.-Y., Wang, L., Xiao, B., Wang, X.-C. and Yang, Y.J. 2016. Diverse Colletotrichum species cause anthracnose of tea plants (Camellia sinensis (L.) O. Kuntze) in China. Sci. Rep. 6: 35287

Wang, X., Liu, X., Wang, R., Fa, L., Chen, L., Xin, X. et al. 2021. First report of Colletotrichum aenigma causing walnut anthracnose in China. Plant Dis. 105: 225.

Weir, B. S., Johnston, P. R. and Damm, U. 2012. The Colletotrichum gloeosporioides species complex. Stud. Mycol. 73: 115-180. 
White, T. J., Bruns, T., Lee, S. and Taylor, J. 1990. Amplification and direct sequencing of fungal ribosomal RNA genes for phylogenetics. In: PCR Protocols: A Guide to Methods and Applications, eds. by M. A. Innis, D. H. Gelfand, J. J. Sninsky and T. J. White, pp. 315-322. Academic Press, San Diego, CA, USA.
Yan, J.-Y., Jayawardena, M. M. R. S., Goonasekara, I. D., Wang, Y., Zhang, W., Liu, M. et al. 2015. Diverse species of Colletotrichum associated with grapevine anthracnose in China. Fungal Divers. 71: 233-246. 